\title{
DYNAMIC JOINT STIFFNESS AND CO-CONTRACTION IN SUBJECTS AFTER TOTAL KNEE ARTHROPLASTY
}

\author{
by
}

Kevin M. McGinnis

\begin{abstract}
A thesis submitted to the Faculty of the University of Delaware in partial fulfillment of the requirements for the degree of Master of Science in Biomechanics and Movement Science
\end{abstract}

Fall 2011

Copyright 2011 Kevin M. McGinnis

All Rights Reserved 


\title{
DYNAMIC JOINT STIFFNESS AND CO-CONTRACTION IN SUBJECTS AFTER TOTAL KNEE ARTHROPLASTY
}

\author{
by \\ Kevin M. McGinnis
}

Approved:

Lynn Snyder-Mackler, Sc.D.

Professor in charge of thesis on behalf of the Advisory Committee

Approved:

Charles Swanik, Ph.D.

Director of the Biomechanics and Movement Science Program

Approved:

Kathleen S. Matt, Ph.D.

Dean of the College of Health Sciences

Approved:

Charles G. Riordan, Ph.D.

Vice Provost for Graduate and Professional Education 


\section{ACKNOWLEDGMENTS}

I'd like to thank my parents Mark and Pat, and my brother Brian for their continued support and encouragement throughout my work.

I am grateful to Lynn for her invaluable guidance and insight. I am also grateful to Joe for his insight and patience.

I'd like to acknowledge everyone in Lynn's lab as well as the other labs in the MAL; their advice and encouragement will always be appreciated. 


\section{TABLE OF CONTENTS}

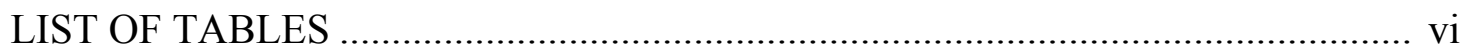

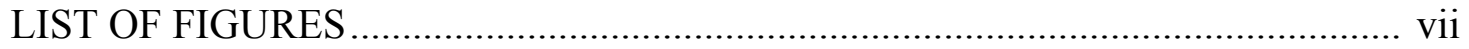

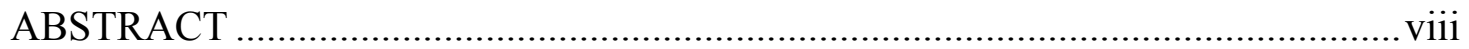

Chapter

1 INTRODUCTION …………………………………………………... 1

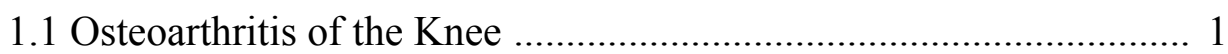

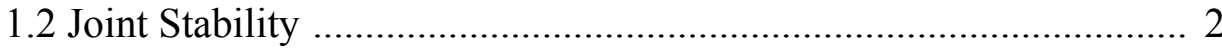

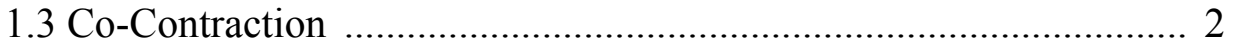

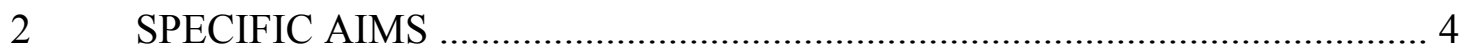

3 ALTERED MECHANICS IN THE ANKLE KNEE AND HIP ...................... 7

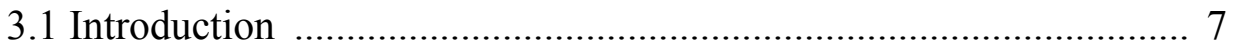

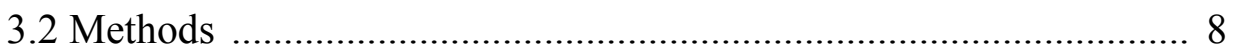

3.3 Results ............................................................................. 13

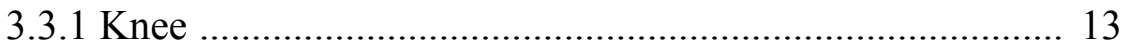

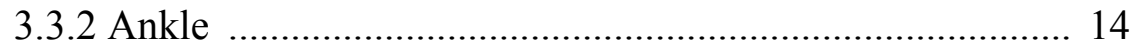

3.3.3 Hip ............................................................................. 15

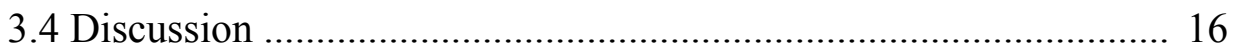

4 CO-CONTRACTION IN THE ANKLE AND KNEE................................. 20

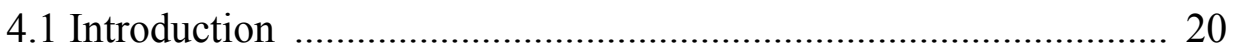

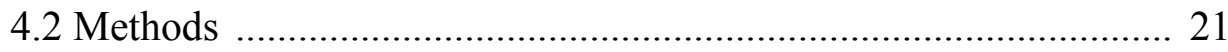

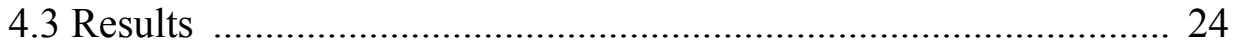

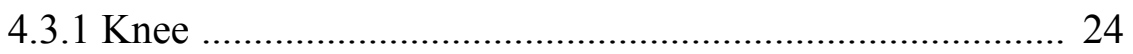

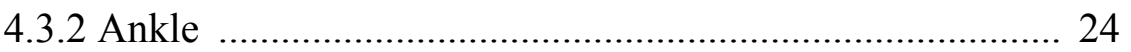

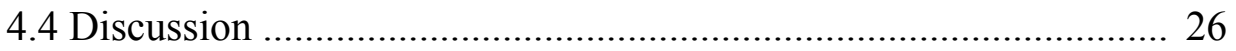


5.1 Aim 1 28

5.2 Aim 2 28

5.3 Clinical Significance 29

5.4 Future Directions

REFRENCES .

30

Appendix

HUMAN SUBJECTS REVIEW BOARD APPROVAL 33 


\section{LIST OF TABLES}

Table $3.1 \quad$ Subject Demographics............................................................... 9 


\section{LIST OF FIGURES}

Figure 3.1 Linear region during weight acceptance in which dynamic knee joint stiffness is measured.

Figure 3.2 Linear region during stance in which dynamic ankle joint stiffness is measured.

Figure 3.3 Linear region during stance in which dynamic hip joint stiffness is measured.

Figure 3.4 Knee stiffness, excursion, and peak extension moment split by group and limb.

Figure 3.5 Ankle stiffness, excursion, and peak dorsiflexion moment split by group and limb.

Figure 4.1 Muscle activation and co-contraction in the operated limb of a TKA subject during weight acceptance.

Figure 4.2 Peak co-contraction in the ankle split by group and limb. 25

Figure 4.3 Average co-contraction in the ankle split by group and limb. 25 


\begin{abstract}
Background

Altered gait patterns and movement strategies that exist before total knee arthroplasty (TKA) can persist after surgery and can be detrimental to the prosthesis and the other joints in the leg. The purpose of this study was to examine dynamic joint stiffness and co-contraction in the lower limbs joints after total unilateral knee arthroplasty.

\section{Methods}

A cross-sectional design was used with 36 subjects at 6 months after TKA, 48 subjects at 1 year after TKA, and 22 healthy controls. Joint excursions, peak joint moments, and co-contraction across the joints were measured. Dynamic joint stiffness was calculated as the moment divided by the angle during gait. Kinematic and kinetic data were collected using 2 in-ground force plates and an 8-camera motion capture system. EMG data was collected using 16 surface electrodes on the lower limb muscles. Repeated measures ANOVAs were used to compare differences between groups (6 month, 1 year, and control) and between limbs (operated and non-operated). Correlations between joint stiffness and co-contraction were also calculated.

\title{
Findings
}

Stiffness was higher in the operated knee in the 6 month group as well as in the operated $\operatorname{limb}(p=0.002, p=0.016)$. There was also a significant group by limb interaction effect $(\mathrm{p}=0.022)$. No significant differences were found in co-contraction between groups at the knee. Ankle stiffness was lower in the 1 year group $(p=0.001)$. 
Co-contraction levels across the ankle were also significantly higher in the 6 month and 1 year groups $(\mathrm{p}<0.001, \mathrm{p}<0.001)$ compared to controls as well as in the operated $\operatorname{limb}$ compared to the non-operated $\operatorname{limb}(\mathrm{p}=0.019)$.

\section{Interpretation}

The knee experienced decreased excursion and quadriceps weakness as well as increased stiffness. Co-contraction was increased at the ankle likely due to the increased moment production. Co-contraction and stiffness were not significantly positively correlated, telling us that the change at the ankle is more likely a mechanical compensation than a compensation for instability. 


\section{Chapter 1}

\section{INTRODUCTION}

\subsection{Osteoarthritis of the Knee}

Osteoarthritis (OA) is a degenerative disease affecting the articular cartilage, most often occurring in the knee ${ }^{1}$. OA is debilitating and causes pain, decreased range of motion and reduced functional performance (Jacobs et al., 2009). Over 500,000 TKA surgeries are performed every year in the United States alone, and that number is expected to reach 3,000,000 per year by 2030 (Kurtz et al., 2007). With this large increase, it is even more important to understand the effect of the knee replacement on every day activities and quality of life. The purpose of knee replacements is to reduce pain and to allow the patient to return to normal patterns of

movement. A non-random progression of OA to the contralateral knee as well as both hip joints has been documented (Milner, 2009). One year after TKA, patients continue to demonstrate significantly worse functional performance during walking and stair climbing which may be related to abnormal movement patterns that increase loading on the non-operated side and attenuate normal joint motions on the operated side (Walsh et al., 1998). If altered gait mechanics persist after surgery, it can lead to the breakdown of the prosthesis and/or OA in the other knee or lower extremity joints. After performing a systematic review of the literature, Milner (2009) determined that future studies should focus on the biomechanics of the hip and ankle joints, as well as the non-operated limb for patients after total knee arthroplasty. 


\subsection{Joint Stability}

Joint stability is crucial in order for patients to maintain a high quality of function. In a study of patients with knee OA, (Fitzgerald et al., 2004) found that $63 \%$ of their subjects reported knee instability (the knee buckling or 'giving way') and that $44 \%$ of these subjects reported that it affected their ability to function. The perception of joint instability persists after TKA and can negatively affect function and joint health ([Barsoum et al., 2011] and [Lo et al., 2010]). Improving joint stability should be addressed in rehabilitation programs in addition to methods to counteract pain and muscle weakness.

Knee stiffness is an important factor in joint stability and creating a 'stiffer' knee through alterations in muscle coordination patterns or biomechanical compensations could be one way individuals function in the presence of knee instability during gait. Knee stiffness is a function of the forces at the knee as well as the range of motion of the knee while these forces are applied. Studies show that after TKA patients have decreased knee flexion and excursion during weight acceptance (Milner, 2009). This decreased excursion would cause an increase in knee stiffness and could also cause the person to alter the mechanics of the hip and ankle joints. An increase in knee joint stiffness may result in an increase in the compressive forces and loading rates at the knee.

\subsection{Co-Contraction}

Another common change in the gait of people with OA is altered muscle

activity patterns. Reduced knee excursion and increased activity of knee flexors and extensors during stance is a common strategy to compensate for pain and knee instability (Rudolph et al., 2001). Increased co-contraction of antagonist muscles 
crossing the knee reduces shear forces; however, this increases compressive forces causing a stiffer knee environment (Rudolph et al., 2001). Co-contraction also increases metabolic demand during walking due to increased muscle activity. Because pre-surgery gait patterns can persist up to 18 months past TKA (Milner, 2009), gait patterns post-TKA must be empirically evaluated to determine if detrimental patterns such as decreased knee excursions and co-contraction are present. Increased cocontraction is one mechanism that may result in increased dynamic joint stiffness during gait. It is important to evaluate because if we know the cause of the stiffer knee we can develop rehabilitation strategies to address it and normalize movement patterns. 


\section{Chapter 2}

\section{SPECIFIC AIMS}

Knee Osteoarthritis $(\mathrm{OA})$ is a degenerative disorder that can dramatically impair a person's ability to take part in daily activities. Total knee arthroplasty (TKA) is a widely used procedure in the management of OA in the knee. Over 500,000 TKA operations are performed each year, comprising nearly half of all total joint arthroplasties (Jacobs et al. 2009). Return to normal gait is the main goal for patients after surgery (McClelland et al., 2007). However, abnormal movement patterns persist after TKA and these biomechanical alterations result in excessive loads on the operated and non-operated limb (Milner, 2009). Increased compressive forces and loading rates may damage the cartilage of the non-operated knee as well as the components of the joint prosthesis in the operated knee. Therefore, it is critical that we clarify which specific biomechanical abnormalities contribute to the abnormal movement patterns.

Joint stability is the ability of a joint to maintain a specific angle or velocity depending on the action being performed. Before and after TKA, joint stability is reduced ([Barsoum et al., 2011] and [Lo et al., 2010] and [Mündermann et al., 2005]) likely as a result of pain, swelling, and quadriceps weakness. Joint stiffness and proprioception are important factors in determining the stability of a joint. Dynamic joint stiffness (DJS) is the ability of muscles, bones, and other soft tissues to resist displacement given an external moment. DJS is a useful tool in analyzing how a 
motor task is coordinated at a joint level, how stable a joint is and what adaptations are being made to improve performance or prevent injury.

DJS is defined as the change in net moment at a joint divided by the change in joint angle (Zeni and Higginson, 2009). Increased peak joint forces and loading rates, variables associated with DJS, would result in a higher joint stiffness. While increased DJS may improve the stability of the joint, it may also have negative consequences on the integrity of the articular cartilage and the joint prosthesis. Higher levels of stiffness may accelerate the progression of OA through an increase in joint compressive forces and loading rates. Reduced knee excursion is one of the most frequently observed characteristics of gait seen in individuals with knee OA and may result in elevated knee stiffness.

It is important to determine the specific variables that result in an increase in DJS in order to create rehabilitation regimens to address the abnormalities. Additionally we sought to determine the relationship between co-contraction and DJS. If a significant correlation exists, rehabilitation regimens can be created to address the increase in co-contraction as well.

The purpose of this study was to analyze DJS in the ankle, knee, and hip joints compared to control subjects without knee OA and to determine the level of antagonist muscle co-contraction in the ankle and knee joints.

The specific aims are as follows:

1) Determine the changes in hip, ankle and knee stiffness during walking, for operated and non-operated limbs, in unilateral TKA subjects at six months and one year after TKA, compared to healthy controls. 
Hypothesis 1.1 - Subjects at six months after TKA will have higher DJS in the operated limb compared to the non-operated limbs and compared to controls

Hypothesis 1.2 - Stiffness will decrease between 6 months and 1 year after TKA in the operated limb compared to the non-operated limbs and compared to controls

2) Analyze the level of co-contraction at the knee and ankle, and determine the correlation between DJS and co-contraction in unilateral TKA subjects during walking, compared to healthy controls.

Hypothesis 2.1 - Subjects at 6 months after TKA will have higher levels of co-contraction at the knee and ankle of the operated limb compared to controls

Hypothesis 2.2 - There will be a significant positive correlation between co-contraction and joint stiffness in the knee and ankle joints 


\section{Chapter 3}

\section{ALTERED MECHANICS IN THE ANKLE, KNEE AND HIP}

\subsection{Introduction}

The main goals after TKA are to relieve pain and to return to normal gait mechanics in the lower limbs. Reduced stance time and reduced loads (net moments and vertical ground reaction forces) on the painful leg, as well as increased loads and demand on the non-painful leg are typical of the gait pattern prior to TKA. Also, muscle co-contraction is increased on the painful leg prior to TKA. Altered gait patterns before TKA can persist after surgery and can be detrimental to the prosthesis and the other joints in the leg. It is important to study kinematics with respect to the moments acting about the joint in order to understand the causes of the changes observed.

Muscle weakness, especially in the quadriceps, is a frequent problem in subjects after TKA. In the presence of persistent muscle weakness after TKA, subjects may adopt altered movement strategies such as decreased range of motion and increased co-contraction during gait. Knee instability is another problem after TKA and can significantly affect a person's ability to perform common daily activities. Previous work has shown reduced knee excursions as well as an increase in knee stiffness after TKA but has not explained if these are related (McGinnis et al., 2010). Also, it is not known if increased co-contraction after TKA is related to increased stiffness or other mechanical changes. 
The purpose of this aim is to study the DJS of the hip, knee, and ankle after TKA and to determine the cause of any changes in stiffness and the effects these changes have on the gait pattern. Our hypothesis is that there will be an increase in DJS in the operated limb at 6 months after TKA compared to controls and that this will decrease to the level of controls at 1 year after TKA.

\subsection{Methods}

\section{Subjects}

The design for this study was cross-sectional and includes 36 subjects six months after unilateral TKA and 48 subjects one year after unilateral TKA (See Table 3.1, values represent means \pm standard deviations). 22 healthy adults with no reported knee pain comprised the control group for this study. Healthy control "operated" limbs were randomly selected between left and right limbs, and matched the percent of knee replacements between the left and right limbs in our subject pool. All subjects signed informed consent forms prior to participation in the study and were allowed to withdraw from the study at any point for any reason. Subjects were excluded if they had a self-reported level of pain greater than or equal to $4 / 10$ in the non-operated limb, neurological or vascular problems that interfere with their ability to perform the ascribed tasks, diabetes that impairs lower extremity sensation, or are currently receiving treatment for cancer. 
Table 3.1 Subject demographics.

\begin{tabular}{lll}
\hline & TKA Subjects & Control Subjects \\
\hline Gender & 41 male 41 female & 12 male 12 female \\
Age $(\mathrm{yr})$ & $66.87 \pm 7.93$ & $62.65 \pm 6.56$ \\
Height $(\mathrm{m})$ & $1.70 \pm 0.104$ & $1.72 \pm 0.108$ \\
BMI $\left(\mathrm{kg} / \mathrm{m}^{2}\right)$ & $30.65 \pm 5.17$ & $28.85 \pm 4.98$ \\
\hline
\end{tabular}

\section{Motion Analysis}

Joint kinematics and kinetics during gait were measured using an 8camera motion capture system (VICON, Oxford Metrics Ltd., London, UK) synchronized with two force plates (Bertec Corporation, Worthington, $\mathrm{OH}$ ). Retroreflective markers were placed bilaterally on anatomical landmarks and rigid thermoplastic shells were placed on the thigh, shank and pelvis. Subjects walked at a self-selected pace. 6-7 walking trials were collected and the average of these trials was used in the analysis. Joint angles were calculated using Euler angles. Joint moments were calculated using inverse dynamics techniques, and the moments were normalized to subject height and weight using Visual 3D software (C-motion, Germantown, MD). The time points between consecutive heel strikes were normalized to 100 points. 


\section{Dynamic Joint Stiffness}

Dynamic joint stiffness was defined as the change in moment divided by the change in angle:

$$
D J S=\frac{\Delta M}{\Delta \theta}
$$

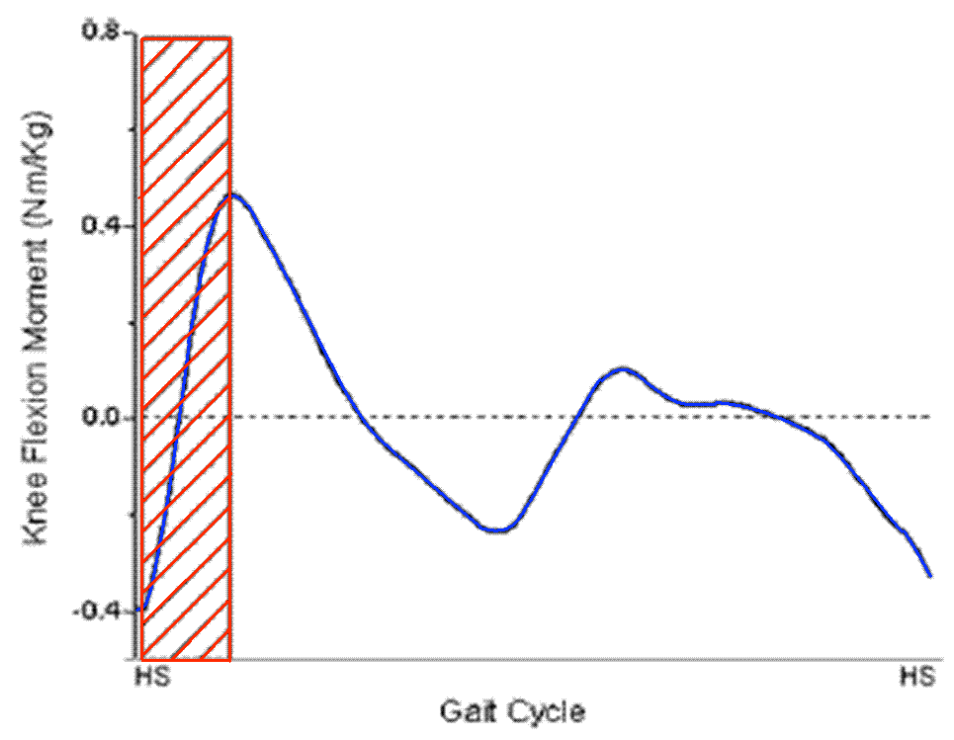

Figure 3.1 Linear region during weight acceptance in which dynamic knee joint stiffness is measured.

The joint moment was plotted against the knee angle and a linear fit of the slope was determined to be the joint stiffness. For the knee, this was done during weight acceptance which was determined to be the linear region in which the average external knee flexion moment starts to increase and ends with peak knee flexion (Fig. 3.1) (Zeni and Higginson, 2009). 


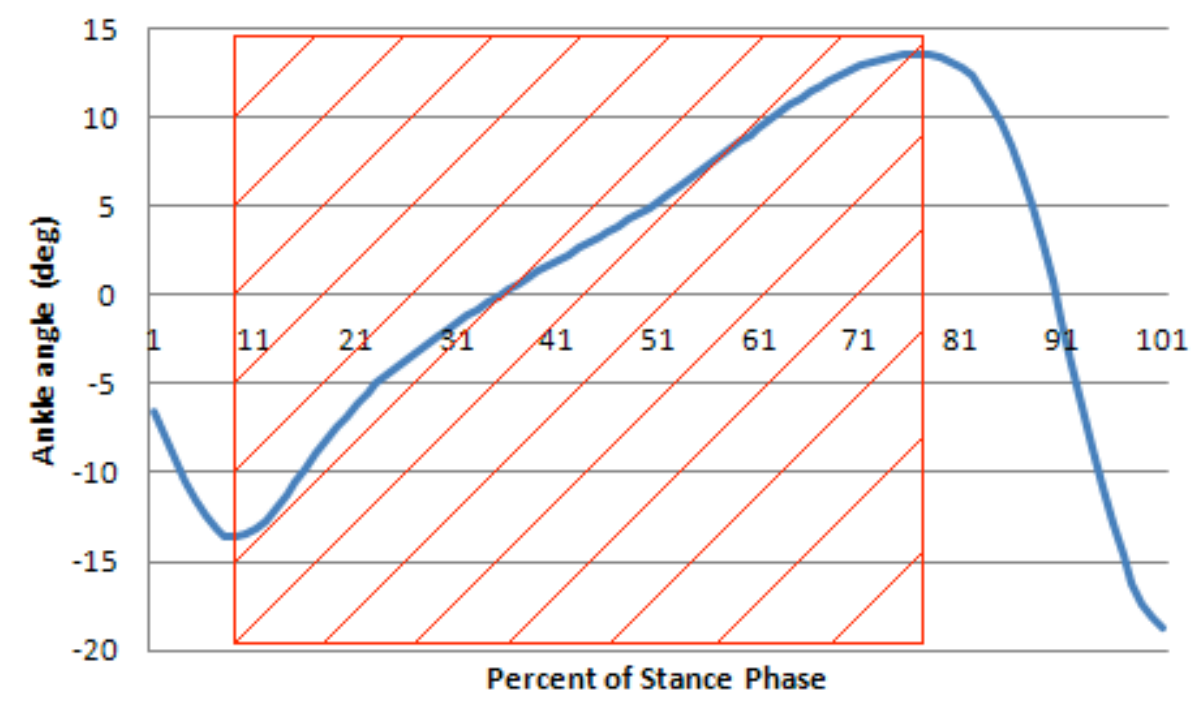

Figure 3.2 Linear region during stance in which dynamic ankle joint stiffness is measured.

For the ankle, stiffness was calculated during the linear region beginning with maximum plantarflexion and ends at maximum dorsiflexion (Fig. 3.2). Hip stiffness was calculated from minimum to maximum hip flexion (Fig. 3.3). These phases were chosen because they encompass important parts of stance phase. For the knee the phase chosen was weight acceptance and for the ankle and hip the phases include the shift between weight acceptance and push-off. 


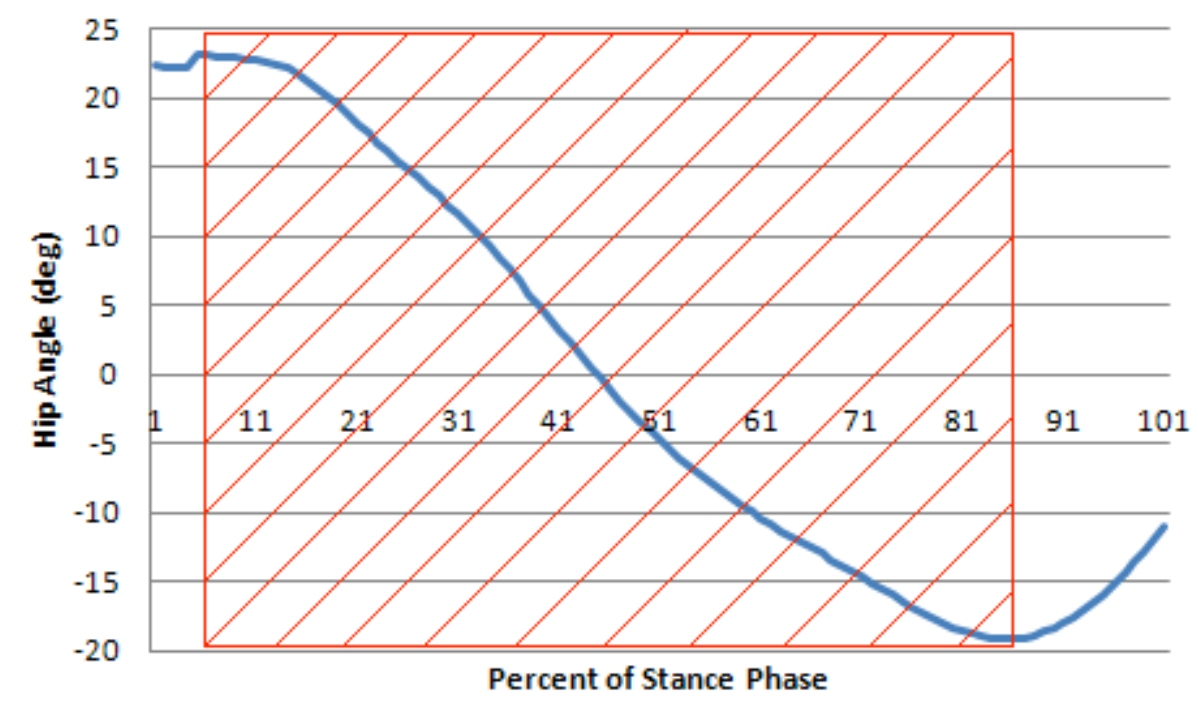

Figure 3.3 Linear region during stance in which dynamic hip joint stiffness is measured.

\section{Excursion and Peak Moments}

Excursion for all joints were calculated as the range of motion during the aforementioned phases. Peak moments were determined as the maximum moments during the aforementioned phases.

\section{Statistical Analysis}

Repeated measures ANOVAs were run (PASSW 18.0, SPSS Inc.) to compare joint stiffness, joint excursion, and peak moments between limbs and between groups. There were three groups: 6 months after TKA, 1 year after TKA and control subjects. Each subject had one repeated measure: operated and non-operated limbs. Separate repeated measures ANOVAs were run for each joint: knee, ankle, and hip. P-values equal to or less than $\mathrm{p}=0.05$ were considered significantly different. Post-hoc testing was done when necessary. 


\subsection{Results}

\subsubsection{Knee}

Subjects at 6 months had higher knee stiffness compared to subjects at 1 year $(\mathrm{p}=0.002)$ when compared by group (Fig 3.4). Differences in knee stiffness between 6 months and controls approached significant levels $(p=0.065)$. Knee stiffness was higher in the operated knee compared to the non-operated knee $(p=0.016)$, and there was a significant limb by group interaction effect $(p=0.022)$.

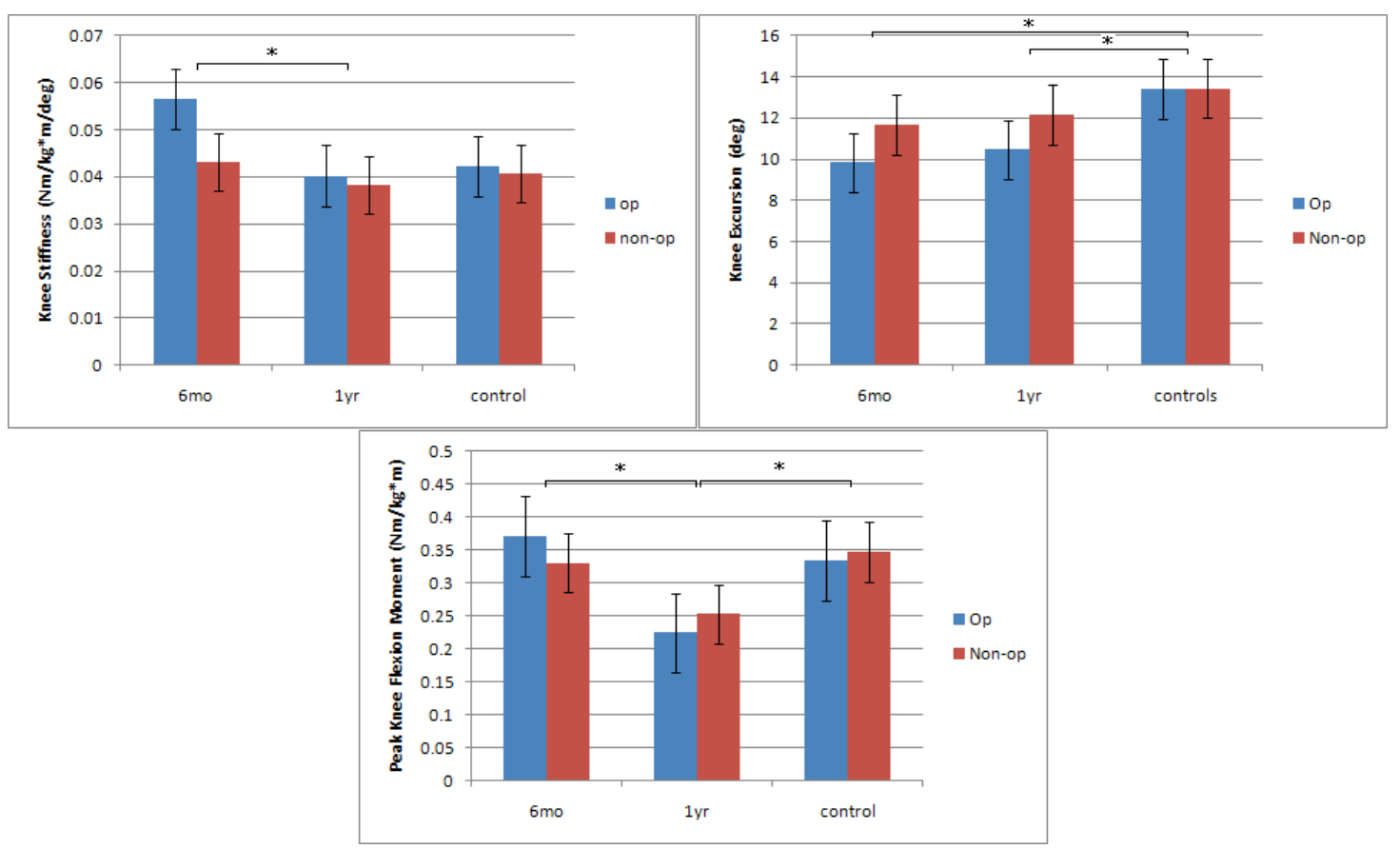

Figure 3.4 Knee stiffness, excursion, and peak extension moments split by group and limb. Asterisk (*) denotes significant difference between groups. Significant differences also exist between limbs for stiffness and excursion.

Peak knee flexion moment was lower at 1 year compared to 6 months and control groups $(\mathrm{p}<0.001, \mathrm{p}=0.006)$ (Fig 3.2). There was no significant difference 
found between the 6 month and control groups and no significant difference existed between limbs for peak flexion moment.

Knee excursion was lower at 6 months $\left(10.76^{\circ}\right)$ and 1 year $\left(11.30^{\circ}\right)$ compared to controls $\left(13.43^{\circ}\right),(\mathrm{p}=0.008, \mathrm{p}=0.025)$. Subjects at 6 months went through less excursion than those at 1 year; however this difference was not significant. There was a difference between limbs in excursion with the operated limb being lower than the non-operated limb $(\mathrm{p}=0.004)$. In the 6 month and 1 year groups, the excursion of the non-operated limb was roughly 2 degrees higher than in the operated limb.

\subsubsection{Ankle}

Subjects 1 year had lower ankle stiffness compared to controls $(\mathrm{p}=0.001)$ (Fig 3.5). Subjects at 6 months had higher ankle stiffness than at 1 year; this difference was not significant but approached significant levels $(\mathrm{p}=0.063)$. There was no significant difference found between limbs for stiffness.

Peak ankle dorsiflexion moment was lower at 6 months and 1year compared to controls $(\mathrm{p}=0.007, \mathrm{p}=0.001)$. There was no significant difference found between 6 months and 1 year or between operated and non-operated limbs for peak dorsiflexion moment.

Ankle excursion was higher 1 year compared to controls $(\mathrm{p}=0.019)$. There was no significant difference found between 6 months and 1 year or between operated and non-operated limbs for excursion. 


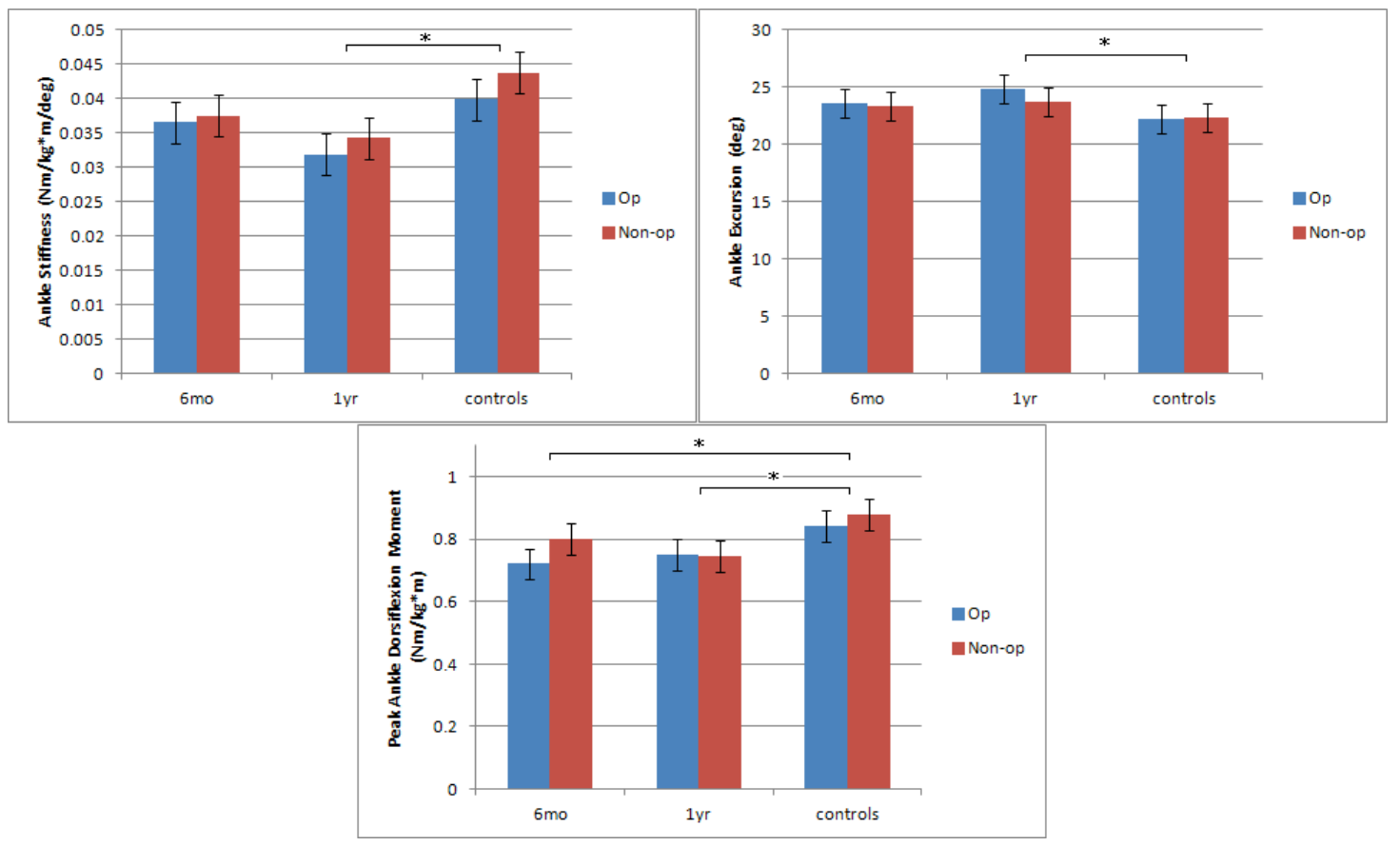

Figure 3.5 Ankle stiffness, excursion, and peak dorsiflexion moments split by group and limb. Asterisk (*) denotes significant difference between groups.

\subsubsection{Hip}

No significant difference was found between groups for hip stiffness, although stiffness was higher in the non-operated limb compared to the operated limb $(\mathrm{p}=0.002)$.

Peak hip extension moment was lower at 1 year compared to 6 months and controls $(\mathrm{p}=0.03, \mathrm{p}=0.041)$. There was no significant difference found between 6 months and controls. Peak extension moment was lower in the operated limb compared to the non-operated $\operatorname{limb}(\mathrm{p}=0.002)$. 
Hip excursion was lower at both 6 months and 1 year compared to controls $(\mathrm{p}<0.001, \mathrm{p}<0.001)$. There was no significant difference found between operated and non-operated limbs for excursion.

\subsection{Discussion}

Our results support our hypothesis that subjects after TKA initially have greater stiffness in the operated knee. For the knee, subjects had greater stiffness 6 month after surgery but at 1 year there was no difference between limbs or compared to the control group. Our hypothesis was that subjects would have higher stiffness in the operated limb compared to the non-operated limb. This however, was not the case for the ankle and hip joints. In the ankle the 1 year group showed a decrease in stiffness compared to controls, whereas the 6 month group showed no differences compared to controls. The hip showed no differences in stiffness between groups. There is a difference between limbs at the hip; however, this difference is due to an increase in stiffness of the control group's "non-operated" limb and is not likely to be a real difference in the patient population.

Lower limb stability and balance is important in avoiding falls; however, the elderly have a decreased ability to regain balance after a perturbation (Arampatzis et al., 2008). This is related to a decline in muscle strength and a decrease in rate of force generation (Arampatzis et al., 2008). We know that subjects after TKA have further weakness in the quadriceps muscle of the operated limb and that this weakness is correlated with poor functional performance (Mizner and Snyder-Mackler, 2005). The increase in stiffness reduces the normal shock absorbing mechanism of the lower extremity during every step. This may be related to the typical loss of strength that we see after TKA. Previous work has shown a shift in support moment away from the 
knee and toward the other lower extremity joints in patients with OA prior to TKA ([Zeni and Higginson, 2009] and [Yoshida et al., 2008] and [Mandeville et al., 2007]).

Knee joint excursion was lower in the 6 months group compared to controls and is lower in the operated limb as well. Decreased knee excursion is common after TKA and may be related to an increase in pain and decreased muscle weakness (Milner 2009). Peak knee extension moment is lower in the 1 year group from the level of the 6 month and control groups. The decreased excursion in the operated knee of the 6 month group is likely the cause of the increased stiffness observed. The knee excursion is decreased in the operated knee of the 1 year group, which would cause an increase in stiffness, however, the extension moment is also decreased which causes the stiffness in the operated knee of the 1 year group to remain at the level of controls.

The data we collected suggest that the ankle is used to compensate for the mechanical changes at the knee. The ankle is less stiff at 1 year compared to controls. The cause of this decreased ankle stiffness is an increase in ankle excursion and a decrease in ankle moments in the 1 year group. Ankle joint excursion is increased at 1 year $\left(24.30^{\circ}\right.$ compared to $22.25^{\circ}$ for controls). There are no limb differences at the ankle for stiffness, peak moments, or excursion, which tells us that both ankles are utilized equally to compensate for altered knee mechanics.

Mündermann et al. (2005) determined there were increased loading rates at all of the lower limb joints in subjects with medial compartment knee OA. These changes were due to alterations in gait due learned to cope with knee pain. The increase in stiffness can occur as a result of an increase in loading rates. The knee no longer acts like a normal shock absorber and therefore is subject to higher loads and 
loading rates. We know that abnormal movement patterns learned before TKA can persist post-TKA and can be detrimental to the joints of both limbs (Walsh et al. 1998). Mündermann et al. (2005) also found no differences in sagittal plane ankle moment in subjects with knee OA. This suggests that the increase in ankle moment and ankle stiffness we see post-TKA is the result of a compensation strategy employed after knee replacement.

Shifting the load away from the involved knee and reducing the shock absorbing function of the quadriceps, coupled with an increase in knee stiffness may have a detrimental effect at the other lower extremity joints. Increased loading rates and peak joint moments can be harmful to the other joints of the lower limbs. Studies should that quadriceps weakness is correlated with poor functional performance and strengthening of the quadriceps muscles may be beneficial to overall joint health and function (Mizner and Snyder-Mackler, 2005). The peak ankle moments occur during push-off in stance phase which suggests the subjects are using the ankle to push off during the end of stance. The use of the ankle as compensation for deficits may lead the subject to rely less on the knee joint as well as the quadriceps, hamstrings, and other muscles.

Some limitations of the study include our inability to directly measure the stability of the joints or determine the underlying causes of the changes in gait mechanics. Some questions we are unable to answer are: what is the cause of stiffness? Is it co-contraction? Does it occur only in subjects with quadriceps weakness after TKA? Does it more often affect subjects who have feelings of instability? Future work that is able to answer these questions will lead to 
rehabilitation strategies that can be implemented to normalize movement patterns after TKA. 


\section{Chapter 4}

\section{CO-CONTRACTION IN THE ANKLE AND KNEE}

\subsection{Introduction}

Patients with knee OA typically demonstrate altered muscle coordination patterns during gait (Heiden at al., 2009). Reduced knee excursion and increased activity of knee flexors and extensors during stance is a common strategy to compensate for pain and knee instability in patients with knee OA. Increased levels of co-contraction may affect joint kinetics and kinematics and may also cause an increase in dynamic joint stiffness and joint compression forces. Patients post-TKA also ambulate with reduced knee flexion excursion which can lead to asymmetrical movement patterns and can possibly be detrimental to the prosthesis. We do not know if co-contraction that is present before TKA continues after surgery or if increased cocontraction is related to increases in DJS. Because pre-surgery gait patterns can persist up to 18 months after TKA (Walsh, 1998), gait patterns post-TKA must be studied to determine if co-contraction is present. Identifying these abnormal control strategies can lead to the creation of effective interventions for rehabilitation postsurgery.

The purpose of this aim is to study co-contraction in the knee and ankle after TKA. Our hypothesis is that there will be an increase in co-contraction in the operated limb of both the knee and ankle at 6 months post-TKA compared to controls. 
We also hypothesize that there will be a significant positive correlation between knee and ankle stiffness and co-contraction.

\subsection{Methods}

Subjects

The subject pool is the same as described in Chapter 3.

$E M G$

Electromyographic data was collected bilaterally for each subject using active surface electrodes (Motion Lab Systems, Baton Rouge, LA). The skin was cleaned with alcohol prior to electrode placement. Electrodes were placed on the following eight muscles on each limb: gluteus medius, lateral hamstring, vastus lateralis, vastus medialis, tibialis anterior, soleus, and the medial and lateral heads of the gastrocnemius. Prior to walking, maximum volitional isometric contractions (MVICs) were performed to determine the maximum levels of voluntary contraction. During MVICs the patient was asked to contract each muscle group isometrically, with as much force as possible, against the resistance of the investigator. MVICs for different muscle groups were recorded in separate trials, with each trial containing 1-2 seconds in which the muscle is not active.

The EMG signal was pre-amplified at the skin and sampled at a rate of $1080 \mathrm{~Hz}$. Visual 3D software (C-motion, Germantown, MD) was used to filter the signals using a low pass filter at $350 \mathrm{~Hz}$. A linear envelope was created, with a high pass filter cutoff of $20 \mathrm{~Hz}$, and data were normalized to the maximum signal obtained either during MVIC or walking trial. 


\section{Co-Contraction}

Antagonist muscle co-contraction was calculated using the following equation developed by Rudolph et al. (2001).

$$
C C I=\frac{E M G_{S}}{E M G_{L}}\left(E M G_{S}+E M G_{L}\right)
$$

$\mathrm{EMG}_{\mathrm{S}}$ is the signal from the least active muscle while $\mathrm{EMG}_{\mathrm{L}}$ is the signal from the more active muscle. The vastus lateralis and the semimembranosus were used to calculate co-contraction at the knee, and the tibialis anterior and the medial head of the gastrocnemius were used to calculate co-contraction at the ankle. Co-contraction was determined during the same time periods as joint stiffness for the respective joints. Peak co-contraction was determined to be the maximum value during these time periods. Average co-contraction was calculated as the area under the co-contraction curve during these time periods. Figure 4.1 shows a plot of this calculation. 


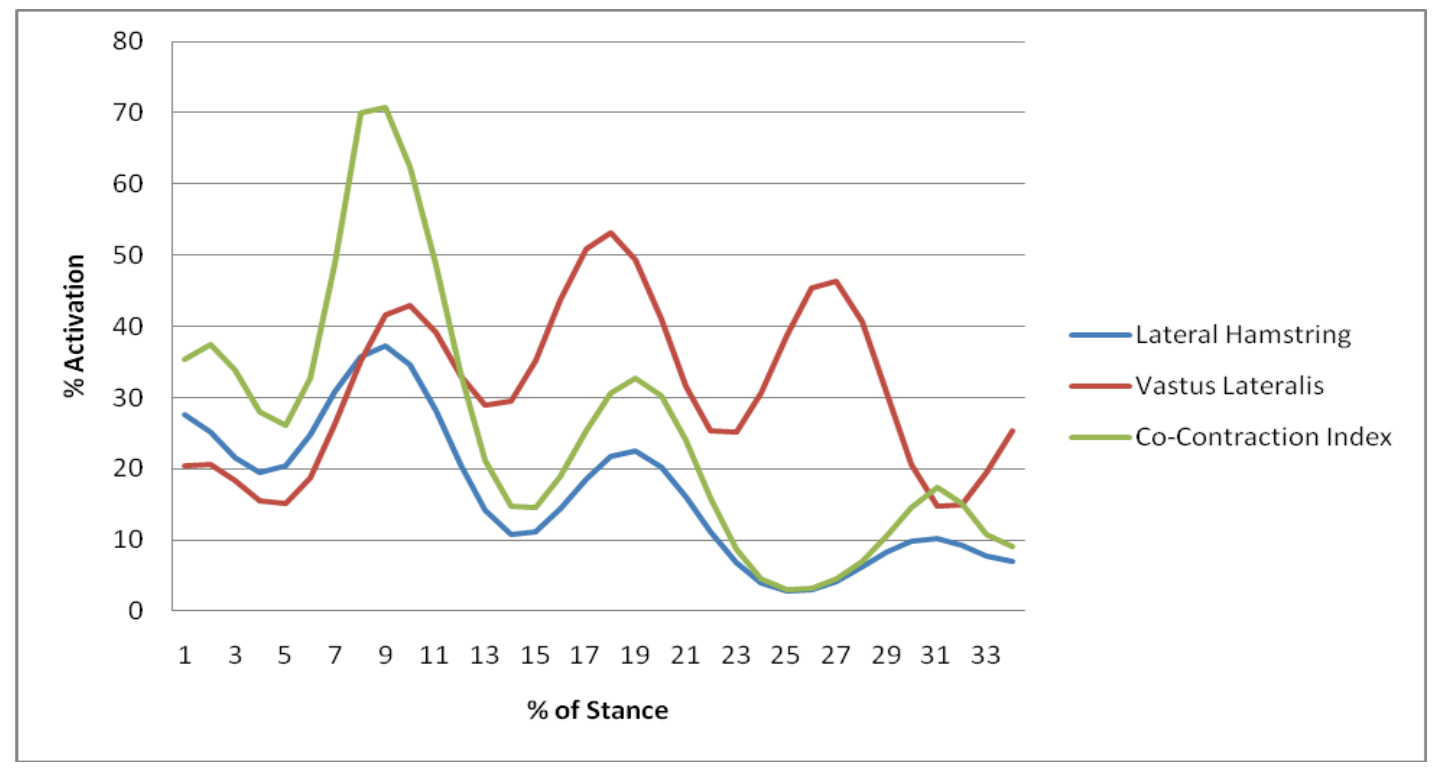

Figure 4.1 Muscle activations and co-contraction in the operated limb of a TKA subject during weight acceptance.

\section{Statistical Analysis}

Repeated measures ANOVAs were run (PASSW 18.0, SPSS Inc.) to compare peak co-contraction and average co-contraction between limbs and between groups. There were three groups: 6 months after TKA, 1 year after TKA and control subjects. Each subject had one repeated measure: operated and non-operated limbs. Separate repeated measures ANOVAs were run for each joint: knee and ankle. Pearson's correlation coefficients were also calculated between stiffness and cocontraction for both the knee and ankle. P-values equal to or less than $p=0.05$ were considered significantly different. Post-hoc testing was done when necessary. 


\subsection{Results}

\subsubsection{Knee}

There were no significant differences between groups or between limbs in either peak or average co-contraction. Also, there was no significant correlation between knee stiffness and either peak $(r=0.225, \mathrm{p}=0.073)$ or average co-contraction $(\mathrm{r}=0.172, \mathrm{p}=0.174)$.

\subsubsection{Ankle}

In the ankle, peak co-contraction was higher 6 months and 1 year after TKA compared to controls $(\mathrm{p}<0.001, \mathrm{p}<0.001)$ (Fig. 4.2). Peak co-contraction was higher at 1 year compared to 6 months; however, this difference was not significant $(p=0.066)$. Peak co-contraction was also higher in the operated limb compared to the non-operated $\operatorname{limb}(\mathrm{p}=0.019)$. Average co-contraction was higher at 6 months and 1 year compared to controls $(\mathrm{p}=0.001, \mathrm{p}<0.001)$. Average co-contraction was also higher in the operated limb compared to the non-operated limb $(p=0.028)$. There was a significant correlation between ankle stiffness and both peak co-contraction $(\mathrm{r}=-$ $0.351, \mathrm{p}=0.04)$ and average co-contraction $(\mathrm{r}=-0.341, \mathrm{p}=0.005)$. 


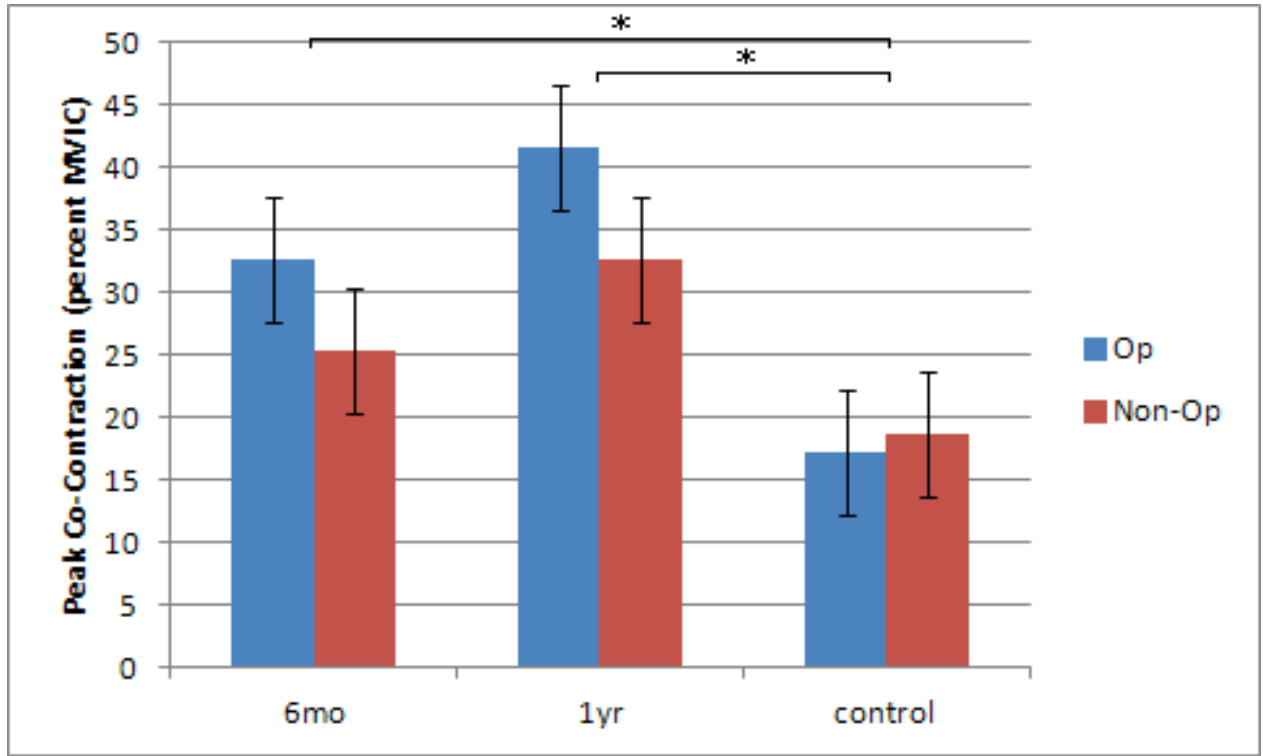

Figure 4.2 Peak co-contraction in the ankle split by group and limb. Asterisk (*) denotes significant difference between groups. Significant differences also exist between limbs.

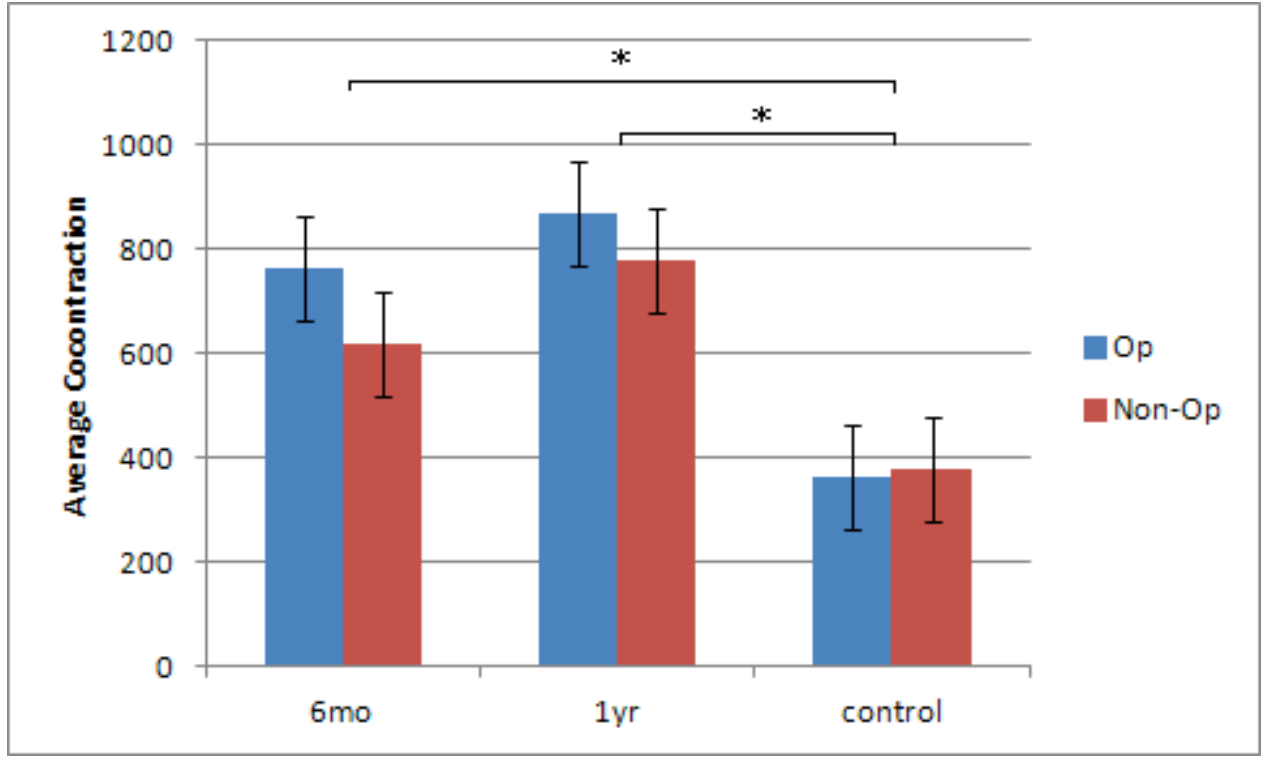

Figure 4.3 Average co-contraction in the ankle split by group and limb. Asterisk (*) denotes significant difference between groups. Significant differences also exist between limbs. 


\subsection{Discussion}

At the knee our results do not support our hypothesis that there will be increased co-contraction and that this increase will be correlated to an increase in stiffness. We anticipated greater co-contraction due to an increase in pre-operative levels but this trend does not continue after surgery. We also expected a relationship between co-contraction and stiffness at the knee. After TKA, there does not appear to be differences in muscle coordination (with respect to co-contraction), but patients still demonstrate attenuated knee excursions. Other factors beyond co-contraction are leading to greater joint stiffness, possibly increased quadriceps activity.

In the ankle, our results partially support our hypothesis. As expected, peak ankle co-contraction and average ankle co-contraction were both significantly higher at 6 months and 1 year compared to controls. These measures were also significantly higher in the operated limb. Also, there was a significant correlation between ankle stiffness and both peak and average co-contraction; however, the correlation was a negative relationship. These findings further demonstrate the ankle's role in compensating for altered knee mechanics. The findings suggest that overall, subjects at 6 months and 1 year use a global co-contraction strategy (average co-contraction) at the ankle, but potentially at one year, subjects also use a more specific co-contraction strategy (peak co-contraction).

The increased co-contraction is most likely a result of increased activation of the plantarflexor muscles for push-off throughout stance phase. The decrease in ankle stiffness at one year is a result of a combination of decreased ankle moment and an increased ankle excursion. The decrease in power absorption of the knee during loading response, due to a stiffer knee with less excursion, may dampen or reduce the ability to generate power at the knee later in stance. Therefore, we require greater 
contributions from other joints, such as the ankle. The ankle compensates for the stiffness and reduced excursion at the knee by increasing its contribution to the propulsion of the lower limb during the stance phase of gait. While the alterations at the knee compensate for instability and quadriceps weakness, those at the ankle are more mechanical in nature. A stiffer ankle, while aiding in compensation for altered knee mechanics, can be detrimental to the health of the ankle joint. Increased peak moments at the ankle and muscle co-contraction across the ankle joint leads to higher compressive forces, which over time can cause a faster progression of $\mathrm{OA}$ in the ankle, tendonopathies, bursitis, and other conditions due to overuse of the joints. If co-contraction at the knee is not the underlying cause of stiffness, we need to investigate other possible causes such as increased muscle activity which is not co-contraction. Rehabilitation strategies specifically designed to reduce cocontraction at the knee post-TKA may not be optimal. 


\section{Chapter 5}

\section{CONCLUSION}

The overall goal of this work was to examine the changes in mechanics at the ankle, knee, and hip as a result of unilateral total knee arthroplasty, specifically by looking at dynamic joint stiffness and co-contraction.

\subsection{Aim 1}

The results from Aim 1 (Chapter 3) show altered mechanics in all of the lower limb joints as late as 1 year after TKA. Joint excursion and peak extension moments are decreased as subjects avoid using the operated knee. The ankle compensates for these changes by increasing plantarflexor activity. These changes, coupled with quadriceps weakness can lead to wear of the prosthesis and break down of the cartilage of other lower limb joints.

\section{$5.2 \operatorname{Aim} 2$}

The results from Aim 2 (Chapter 4) show increased ankle co-contraction at both time points post-TKA. These results agree with the findings in Aim 1 that the ankle is used as the main joint for compensation to altered knee mechanics. Ankle stiffness is negatively correlated with peak and average co-contraction. However, because ankle stiffness and co-contraction are not positively correlated, the ankle is most likely compensating for mechanical deficits by increasing its contributions, whereas the knee may be compensating for muscle weakness, avoidance of use, fear of falling, or continuing learned movement behaviors from before TKA. 


\subsection{Clinical Significance}

Our results showed that after TKA surgery subjects avoid using the knee joint during gait (decreased excursion, increased stiffness) and compensate by using the ankle more (increased excursion, increased co-contraction). These changes are a short-term solution to mechanical problems and are potentially detrimental to the longterm health of the lower limb joints. Because co-contraction may not be the underlying cause of stiffness at the knee, rehabilitation strategies that focus on decreasing co-contraction may not be optimal. Rehabilitation strategies should focus on restoring normal and symmetrical movement patterns, even though these patterns may not result from an increase in co-contraction.

\subsection{Future Directions}

Future work should include analysis of gait further out from surgery than 1 year. We should also investigate other causes of this early stiff knee gait such as muscle weakness, joint instability, perceived instability or avoidance patterns, or proprioceptive deficits post-surgery. Also, interventions could be designed and studied to decrease joint stiffness, increase knee excursion, and promote a more even distribution of support between joints and symmetric distribution of support between limbs. 


\section{REFRENCES}

Arampatzis A., Kiros K., Mademli L., 2008. Deficits in the way to achieve balance related to mechanisms of dynamic stability control in the elderly. Journal of Biomechanics 41, 1754-1761.

Barsoum W., Lee H., Murray T., Colbrunn R., Klika K., Butler S., Van den Bogert A., 2011. Robotic testing of proximal tibio-femoral joint kinematics for measuring instability following total knee arthroplasty. J Orthop Res. 29, 4752.

Fitzgerald G., Piza S., Irrgang J., 2004. Reports of joint instability in knee osteoarthritis: its prevalence and relationship to physical function. Arthritis and Rheumatism 51(6), 941-946.

Heiden J., Lloyd D., Ackland T., 2009. Knee joint kinematics, kinetics, and muscle coordination in the knee osteoarthritis patient gait. Clinical Biomechanics. 24(10), 883-841.

Jacobs J., Andersson G., Bell J., 2009. The burden on musculoskeletal diseases in the United States - Executive summary. Bone and Joint Decade.

Kurtz S., Ong K., Lau E., Mowat F., Halpern M., 2007. Projections of primary and revision hip and knee arthroplasty in the United States from 2005-2030. J. Bone Joint Surg. Am. 89(4), 780-785.

Lo J., Muller O., Dilger T., Wulker N., Wunschel M., 2010. Translational and rotational joint stability in anterior and posterior cruciate-retaining knee arthroplasty. The Knee. doi:10.1016/j.knww.2010.10.009. 
Mandeville D., Osternig L., Chou L., 2007. The effect of total knee replacement on dynamic support of the body during walking and stair ascent. Clinical Biomechanics. (22), 789-794.

McClelland J., Webster K., Feller J., 2007. Gait analysis of patients following total knee arthroplasty: A systematic review. The Knee. 14(4), 253-263.

McGinnis K., Zeni J., Alnadhi A., Snyder-Mackler L., 2010. Dynamic knee joint stiffness and knee joint moments after unilateral TKA. Abstract/Poster presented at 2010 meeting of the American Society of Biomechanics (ASB) in Providence, RI.

Milner C., 2009. Is gait normal after total knee arthroplasty? Systematic review of the literature. J. Orthop. Sci. 14, 114-120.

Mizner R., Snyder-Mackler L., 2005. Altered loading during walking and sts is affected by quadriceps weakness after total knee arthroplasty. J. Orthop. Res. 23, 1083-1090.

Mündermann A., Dyrby C., Andriacchi T., 2005. Secondary gait changes in patients with medial compartment knee osteoarthritis. Journal of Arthritis and Rheumatism. 52(9), 2835-2944.

Rudolph K., Axe M., Buchanan T., Scholz J., Snyder-Mackler L., 2001. Dynamic stability in the anterior cruciate ligament deficient knee. Knee Surg. Sports Traumatol. Arthrosc. 9, 62-71.

Walsh M, Woodhouse L., Thomas S., Finch E., 1998. Physical impairments and functional limitations: a comparison of individuals 1 year after total knee arthroplasty with control subjects. Physical Therapy. 78(3), 248-258. 
Yoshida Y., Mizner R., Ramsey D., Snyder-Mackler L., 2008. Examining outcomes from total knee arthroplasty and the relationship between quadriceps strength and knee function over time. Clinical Biomechanics. (23), 320-328.

Zeni J., Higginson J., 2009. Dynamic knee joint stiffness in persons with a progressive increase in severity of knee osteoarthritis. Clinical Biomechanics. 24(4), 366-371. 
APPENDIX

\section{HUMAN SUBJECTS REVIEW BOARD APPROVAL}

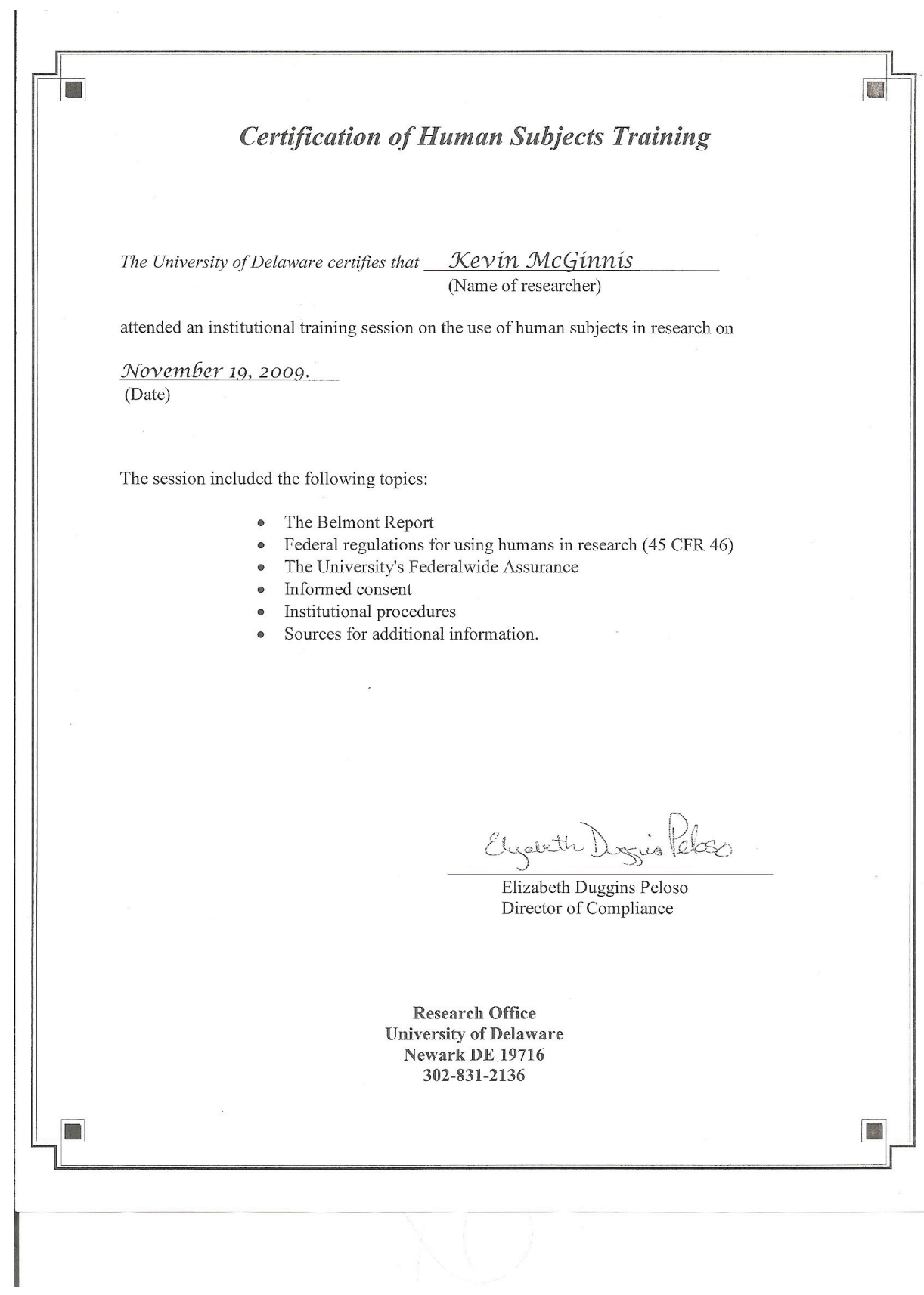

